\title{
Gobierno abierto, un modelo basado en tres pilares: transparencia, rendición de cuentas y participación ciudadana
}

\author{
Open Government, a model based on three pillars: transparency, \\ accountability and citizen participation
}

Lic. Harold Hütt Herrera, M.B.A.

Universidada Estatal a Distancia, Costa Rica

harold.hutt@gmail.com

\section{RESUMEN:}

El presente artículo permite identificar los aspectos conceptuales más relevantes en relación con el modelo de Gobierno abierto, para lo cual se realizó una revisión bibliográfica de las principales posturas en relación con este tema. Uno de los principales hallazgos del trabajo realizado, es que aún no existe un consenso conceptual sobre el particular, pero sí fue posible identificar elementos comunes que pueden ser utilizados como punto de partida, lo que a su vez ha facilitado la adaptación del modelo a las características culturales y políticas de cada país. Los aportes del trabajo son innovadores, dado que es un tema emergente.

\section{ABSTRACT:}

This paper enables to identify the most relevant conceptual aspects in relation with the open Government model. For this purpose, a bibliographic review was performed of the main lines of thought linked to this topic. One of the most important findings of this paper is that there is no conceptual consensus yet on this subject. However, it was possible to identify some common elements that may be used as a starting point, which has also helped adapt the model to the cultural and political characteristics of each country. The contributions of this paper are innovative, since it is an emerging theme.

\section{RESUMO:}

$\mathrm{O}$ presente artigo permite identificar os aspectos conceituais mais relevantes em relação ao modelo Governo Aberto, para o qual foi feita uma revisão bibliográfica das principais posições em relação a este tópico. Um dos principais achados do trabalho realizado, é que ainda não há consenso conceitual sobre o assunto, mas foi possível identificar elementos comuns que podem ser utilizados como ponto de partida, o que, por sua vez, facilitou a adaptação do modelo às características culturais e políticas de cada país. As contribuições do trabalho são inovadoras, pois é um tema emergente.

\section{RÉSUMÉ:}

Cet essai permet d'identifier les plus importants aspects conceptuels liés au modèle de Gouvernement ouvert. A cet effet, une révision bibliographique a été effectuée au sujet des lignes de pensée majeures en rapport à ce thème. L'une des conclusions de ce travail est qu'il n'existe pas encore un consensus conceptuel sur ce sujet. Néanmoins, il a été possible d'identifier des éléments communs qui peuvent être utilisés comme point de départ, ce qui a aussi facilité l'adaptation du modèle à des caractéristiques culturelles et politiques de chaque pays. Les apports de cet essai sont innovants vu qu'il s'agit d'un thème émergent.

PALABRAS CLAVES
LEGITIMIDAD ESTATAL,
GOBERNANZA, ANÁLISIS
COMPARADO, TENDENCIAS
DE GOBIERNO, DATOS
PÚBLICOS

\section{PALAVRAS CHAVES}

LEGITIMIDADE ESTATAL, GOVERNANÇA, ANÁLISE COMPARADA, TENDÊNCIAS DE GOVERNO, DADOS PÚBLICOS

\section{MOTS CLÉS}

LEGITIMITE ETATIQUE, GOUVERNANCE, ANALYSE COMPAREE, TENDANCES DE GOUVERNEMENT, DONNEES PUBLIQUES 


\section{INTRODUCCIÓN}

El modelo de Gobierno abierto ha surgido como un mecanismo idóneo para impulsar una mayor democracia en las naciones; pero, principalmente, para incentivar la participación ciudadana y promover una mayor credibilidad y legitimación de la acción estatal. No obstante, debido a la reciente creación del término, aún no existen consensos sobre la totalidad de sus alcances e implicaciones, aunque sí se cuenta con parámetros generales comunes, los cuales han facilitado la adopción del modelo, a pesar de las diversas características políticas y culturales de los países.

En razón de lo expuesto, el presente artículo realiza un abordaje teórico de los principios y conceptos vinculados al modelo de Gobierno abierto, con el objetivo de identificar elementos comunes que puedan ser utilizados como punto de partida para el análisis de esta nueva forma de administración, cuyo eje de acción se centra en el fortalecimiento de la gestión democrática y la gobernanza.

Los orígenes de este concepto surgieron a finales del siglo XX, cuando la Organización para la Cooperación y el Desarrollo Económico (OCDE), insistió en que las naciones debían buscar mecanismos para incentivar la transparencia, reducir la corrupción y rescatar la credibilidad en las instituciones públicas.

Es a partir de esto que, en el año 2009, el entonces presidente de Estados Unidos, Barack Obama, emitió una serie de directrices para sus agencias gubernamentales, en las cuales solicitaba implementar mecanismos de transparencia y rendición de cuentas, así como de promover la participación ciudadana en la toma de decisiones.

Lo anterior motivó la creación del Open Government Partnership, donde se establecieron los lineamientos y características generales para la adopción del emergente modelo de Gobierno abierto, el cual fue suscrito por diversos países, según se detalla en el presente artículo, y posteriormente se creó, en el año 2016, la Carta Iberoamericana para un Gobierno Abierto, impulsada con el mismo fin.

Autores como Gómez y Gasco (2012), García (2014), Cutberto, Rocha y Martínez (2015) y Quintanilla y Gill (2016), entre otros, han venido analizando las características de este modelo.

No obstante, han sido pocos los esfuerzos identificados en los cuales se procura realizar un análisis comparado de las posturas, con la finalidad de encontrar puntos de consenso, como ha sido el caso de los trabajos realizados por Aucoin (1990), Barria, González y Cisternas (2017) y Conde y Gutiérrez (2014).

De acuerdo con lo expuesto, se ha considerado que este análisis conceptual comparativo genera un aporte innovador, dado que ofrece una compilación sobre las propuestas y características que en general se circunscriben bajo el término de Gobierno abierto, el cual constituye un concepto emergente de reciente adopción por parte de diversos países alrededor del mundo.

\section{DESARROLLO}

A continuación, se abordan algunos elementos conceptuales, con el fin de facilitar la comprensión del concepto de Gobierno abierto y sus implicaciones en materia de gobernanza y gobernabilidad, en el marco de una gestión orientada a impulsar la participación ciudadana.

\section{Gobierno abierto}

Si bien no hay consenso en cuanto a la definición de Gobierno abierto, se puede entender como un paradigma de gestión gubernamental en el cual se incentiva la participación de la sociedad civil en la definición de políticas públicas, impulsando una gestión democratizante por medio de la cual se informa de manera suficiente y oportuna a la población 
sobre la gestión de gobierno y sus resultados, y se le incentiva a brindar aportes para la toma de decisiones, propiciando un esquema de trabajo colaborativo multipartito y multidireccional, tal y como lo anotan Aucoin (1990), Barria, González y Cisternas (2017) y Conde y Gutiérrez (2014).

Por su parte, el Gobierno abierto es definido por Valenzuela y Bojórquez (2014) como un "conjunto de medidas orientadas a impulsar la transparencia y la participación ciudadana como elementos para avanzar en la apertura en la gestión administrativa y política de los asuntos colectivos” (p. 114). Asimismo, Valenzuela y Bojórquez (2014, p. 115) agregan que el Gobierno abierto

Promueve la coproducción de servicios públicos, mediante la participación ciudadana, aportando valor social de la información que produce la apertura gubernamental. El Estado asume en el Gobierno abierto, una función generadora de información y datos que son puestos a disposición de la sociedad civil, sin lo cual no tendría sentido ni dirección el Estado abierto o el Gobierno abierto.

Aunado a lo anterior, estos autores (Valenzuela y Bojórquez, 2014, p. 115) indican que el Estado abierto tiene dos dimensiones:

a) Una horizontal que reconoce la importancia de activar mecanismos tecnológicos que incentiven la transparencia y la participación ciudadana con poderes de la Unión (Ejecutivo, Legislativo y Judicial).

b) Otra dimensión es la vertical, e involucra a los tres órdenes de gobierno (en el caso de México), el federal, el estatal y el municipal, para generar capacidades institucionales y organizativas, que fomentan la esfera relacional del gobierno y sociedad.

Por otra parte, y según explican Valenzuela y Bojórquez (2014, p. 115), ya no solo se trata de:

Abrir las ventanas del Gobierno para dar a conocer lo que hace, sino que se trata de abrir las puertas a los actores sociales para acceder al lugar donde se deciden las agendas de los problemas públicos. Con Estado abierto se transita a un modelo basado en la concepción tradicional de la transparencia, de una visión legalista hacia otra visión más proactiva, focalizada y colaborativa. El uso de la información pública implica el uso de las tecnologías de la información y la comunicación, a través de la participación ciudadana. La creación de valor implica condiciones de apertura donde la información formada con datos, documentos y archivos requiere utilizarse para crear valor. La información por sí misma no genera utilidad social, sino que se complementa por el uso que hacen las organizaciones de la sociedad civil.

Esta definición permite comprender con claridad cuál es el rol de los actores y la codependencia entre Gobierno y sociedad civil. De igual manera, y según agregan Valenzuela y Bojórquez (2014), todo Gobierno que se considere abierto deberá sentar las bases para la "actuación conjunta de políticas, estrategias, compromisos y diversas formas de actuación colaborativa” (p. 115). Esta gestión, desde luego, promueve y fortalece la democracia en su más pura expresión.

Por su parte, Sandoval (2015, pp. 207-209) plantea tres elementos conceptuales básicos que, a su criterio, deben cumplirse en el marco de un paradigma de Gobierno abierto:

a) El derecho del ciudadano para acceder a información de las instituciones públicas. Esta primera perspectiva es citada por el autor en el marco de la rendición de cuentas.

b) El derecho de los ciudadanos a utilizar la información para gestiones propias de su condición de administrado o usuario.

c) Apertura de datos y reutilización de los mismos por parte de los ciudadanos. Esta perspectiva, a diferencia de la primera, plantea la apertura de datos más segregados, para que pueda incentivarse un análisis más profundo y una generación de propuestas por parte de la sociedad civil. Para efectos 
de esta dimensión, el autor destaca la importancia que adquieren las aplicaciones tecnológicas o “apps” en el uso de datos abiertos.

Nuevamente, se desprende de este concepto el hecho de que el vínculo entre administración pública y sociedad civil se torna esencial para incentivar una relación colaborativa multidireccional, activa y viable, por cuanto, si los canales y mecanismos están establecidos, pero el acceso a orientación e información es limitado, serán reducidas las capacidades reales de cogenerar propuestas y soluciones.

Por su parte, García (2014) expone que, como en toda estructura jerárquica, la información es un elemento que no está accesible para todos y que, cuanto más lejos se esté de la cúspide -política, en este caso-, menos acceso habrá a ella. Asimismo, menciona que, de alguna manera, esto da vigencia al pacto social entre administración y administrados, lo cual hace que se guarde con recelo el acceso a ciertos datos.

No obstante, García (2014) agrega que la población ha encontrado en Internet un canal de acceso a información efectivo para satisfacer ciertas necesidades; pero, de alguna manera, constituye un punto de presión para acceder a la información en tiempo real y esto, desde luego, incentiva la generación de una red participativa con un gran potencial para involucrar a los ciudadanos en las actividades gubernamentales.

Destaca García (2014) que los procesos de innovación en las administraciones han venido acompañados de la administración electrónica o del e-government, permitiendo la prestación de servicios de manera electrónica. No obstante, es importante hacer la salvedad de que Gobierno electrónico y Gobierno abierto son conceptos distintos, como se detalla más adelante.

En forma complementaria, García (2014, p. 76) hace una referencia a la declaración de Londres de junio del 2013 sobre datos públicos, que establece:

Los datos públicos abiertos incrementan la transparencia en los gobiernos y sus actuaciones, lo que promueve el ejercicio de la responsabilidad y el buen gobierno, impulsa el debate público y contribuye a la lucha contra la corrupción (...). Los datos públicos constituyen un recurso esencial en la era de la información. La liberación de estos datos a la esfera pública puede mejorar las vidas de los ciudadanos y promover su acceso puede generar innovación, crecimiento económico y creación de nuevos puestos de trabajo. (...) No compartir estos datos con el público constituye una oportunidad perdida.

A partir de lo anterior, es importante lograr una diferenciación asertiva entre lo que es Gobierno electrónico, a través del cual los usuarios pueden realizar trámites y consultas en línea, y el concepto de Gobierno abierto, que es más amplio e integral, como se ha venido detallando, y cómo aportan otros autores que se incluyen en este análisis.

De acuerdo con las fuentes analizadas (Barria, González y Cisternas, 2017; Cutberto, Rocha y Martínez, 2015; García, 2014), tres prácticas principales caracterizan la gestión del Gobierno abierto: transparencia, participación ciudadana y rendición de cuentas.

Estas tres aristas implican una relación activa entre el Gobierno y la sociedad civil, siendo que este vínculo estará determinado en gran medida por la gestión de comunicación, en procura de informar y sensibilizar a la población sobre esta práctica, su relevancia y sus alcances, así como los mecanismos y canales disponibles para la interacción.

Según define Nava en el Diccionario Electoral de Capel (Instituto Interamericano de Derechos Humanos, 2017, p. $492)$,

el concepto de Gobierno abierto involucra varios elementos tales como: la participación ciudadana como eje de acción gubernamental; la transparencia como generadora de insumos para la participación; los datos abiertos como formato de intercambio de información accesible y 
con portabilidad; y las tecnologías de la información y la comunicación (TIC), para potenciar la accesibilidad y la inmediatez y la simplificación administrativa.

Lo anterior se desarrolla para darle respuesta a las exigencias de mayor transparencia, información, rendición de cuentas, orientación y participación ciudadana, eficacia y eficiencia gubernamental, y una efectiva lucha contra la corrupción. Agrega Nava, en el Diccionario Electoral de Capel (Instituto Interamericano de Derechos Humanos, 2017, p. 493), que el Gobierno abierto tiene como objetivo

que la información gubernamental se presente de una manera clara, completa, segura y confiable, institucionalizando una cultura de apertura de la acción de gobierno, y promoviendo la creación de tecnologías para ampliar la comunicación entre este y la ciudadanía, sobre la base de aspectos como la rendición de cuentas, la innovación, la participación ciudadana y la transparencia.

Adiciona que implementar un Gobierno abierto debe suponer un rediseño institucional a gran escala, que abarque al aparato burocrático en su totalidad, impulsando la idea de un Estado abierto o gobernanza abierta, sin limitarla a la administración pública.

A partir de algunos análisis, según denotan Barria, González y Cisternas (2017), se puede concluir que la tendencia del Gobierno abierto pretende dar más que voz al ciudadano, impulsando reformas más profundas. Asimismo, destacan la crítica que se hace hacia la Nueva Gerencia Pública como paradigma de administración impulsor de vínculos con la sociedad civil, que también procura un acercamiento con los grupos organizados, pero desde una perspectiva más superficial.

Considerando todo esto, los autores concluyen que el Gobierno abierto es la consecuencia de dos factores: uno relacionado con la necesidad de encontrar conceptos y enfoques de la política capaces de incentivar la participación ciudadana para mejorar la calidad de los servicios públicos y aumentar los niveles de participación; y el segundo, basado en las posibilidades y potencialidades que ofrecen los avances tecnológicos.

Tal como se ha indicado anteriormente, y al igual que lo anotan Aucoin (1990) y Conde y Gutiérrez (2014), concluyen Barria, González y Cisternas (2017) que, aunque pareciera existir un consenso relativamente general sobre la definición de Gobierno abierto, este concepto sigue estando en proceso de consolidación.

No obstante, se ha considerado que el concepto brindado por Nava en el Diccionario Electoral de Capel (Instituto Interamericano de Derechos Humanos, 2017), adicionando algunos elementos puntuales, logra darle mayor integralidad y asertividad al término, generando así la siguiente definición:

El Gobierno abierto es un modelo que tiene como base la interacción con la ciudadanía en la acción gubernamental, desde tres perspectivas: participación activa de la sociedad civil en la toma de decisiones, transparencia gubernamental y rendición de cuentas. En el marco de la interacción entre estos actores (Gobierno y sociedad civil), las tecnologías de la información y la comunicación juegan un papel fundamental.

Esta interacción permite que la ciudadanía ejerza un rol de contrapeso en la toma de decisiones, lo que a su vez tiene una incidencia positiva en la recuperación de la confianza en la gestión estatal y la legitimación de la institucionalidad.

A continuación, se detallan las principales características y aspectos conceptuales de cada uno de los tres pilares del Gobierno abierto. 


\section{Transparencia}

La gestión de transparencia -como ya se ha indicado anteriormente-, está vinculada a la gestión de Gobierno abierto como una forma de impulsar la rendición de cuentas en los gobiernos y facilitar el acceso de los ciudadanos a información procesada para facultar su participación en la toma de decisiones y el ejercicio de un rol "fiscalizador" en relación con la gestión estatal.

Según puntualiza García (2014, p. 79),

la gestión de las administraciones públicas se encuadra en el marco general del contrato social democrático. Este basa su legitimidad en el poder emanado de la soberanía popular y transmitido a los gobernantes como elementos de representación política, encargados de la acción ejecutiva y sujetos de rendición de cuentas a los ciudadanos.

En esta misma línea y referenciando algunas experiencias obtenidas con procesos de esta naturaleza en el Reino Unido y en Estados Unidos, García (2014, p. 84) destaca los siguientes como los pasos para instaurar un Gobierno abierto:

a) Publicación de la información gubernamental en línea en un plazo de tiempo oportuno, estableciendo una presunción de divulgación para toda la información disponible y realizando esta divulgación en formatos abiertos que permiten su descarga, recuperación, indexación y tratamiento por medio de herramientas comunes de consulta web.

b) Mejora de la calidad de la información pública maximizando calidad, objetividad, utilidad e integridad de la información.

c) Creación e institucionalización de una cultura de Gobierno abierto, incorporando los valores de transparencia, participación y colaboración en las administraciones públicas mediante la implementación de prácticas innovadoras y la experimentación con nuevas tecnologías.

d) Creación de un marco normativo para el aprovechamiento de los nuevos canales de relación entre administraciones y ciudadanos creados por la web social y la web semántica, permitiendo el nacimiento de colaboraciones de alto impacto con investigadores, sector privado y sociedad civil en general.

Asimismo, detalla García (2014, p. 85) que existen algunas recomendaciones generales que están siendo recopiladas para facilitar la implementación de este paradigma (Gobierno abierto), incluyendo dentro de estas, propuestas específicas para la transparencia, divulgación y reutilización de la información:

1. Contenido: Ser tan abierto como sea posible, siendo por defecto divulgada cualquier información que no viole la seguridad pública o la ley; los datos publicados deben gozar de un alto nivel de estructuración o ser documentos de texto plano.

2. Priorización: Buscar el mayor rendimiento posible al costo monetario de la transparencia priorizando en un primer momento la información relativa a influencia en los gobiernos, corrupción y supervisión interna y externa e información obligatoriamente requerida a los cargos electos (patrimonio, participación en consejos de administración, etc.), así como toda la información existente en otros medios, generalmente impresos, o que ya estén siendo recopilados y compartidos con el público por otras vías.

3. Oportunidad: No reaccionar a peticiones, sino divulgar de manera pública con anterioridad. En especial, borradores de normativas en fase de debate y previos a su aprobación.

Adicionalmente, García (2014, p. 85) referencia los principios definidos por la Open Government Foundation, en relación con la información (transparencia): 
1. Gratuidad: La información no es verdaderamente pública si no se encuentra disponible en Internet gratuitamente. Si la información solo puede ser obtenida por requerimiento en un registro público, por vía postal o por personación en las oficinas, la información ha de entenderse que no es accesible, pues supone un costo significativo en tiempo y esfuerzo para el ciudadano.

2. Fuente primaria: La información ha de estar disponible como es recogida en sus fuentes, con el mayor nivel de particularidad disponible y no tratada ni agregada. Si la información se tratara para su presentación en informes o estrategias de comunicación, siempre habrá de estar disponible la fuente de datos original junto con la información modificada.

3. Oportunidad: La información ha de hacerse pública con la mayor premura que sea necesaria para conservar al máximo su valor. Para las cuestiones de actualidad, la información debe ser publicada en el menor plazo de tiempo posible y siempre con el mayor nivel de rigor y calidad para ser útil en el debate público y toma de decisiones, debiendo ponerse de manifiesto cuáles de las entregas suponen actualizaciones de informaciones previas.

4. Accesibilidad: La información ha de estar disponible para el mayor número de usuarios posible y para la mayor gama de usos posibles. Los datos han de entregarse en formatos y protocolos estándares que soporten tanto los usos previstos como los no previstos y que permitan la mayor difusión posible. Si los datos son accesibles a través de interfaces interactivas, en el navegador web han de estar disponibles para su descarga íntegra a través de un proceso automático.

5. Automatización: La información debe estar razonablemente estructurada para permitir su tratamiento automatizado. El formato en que se realice la difusión conlleva consecuencias técnicas para su tratamiento futuro. Cuando se divulguen copias fieles de documentos originales como imagen (pdf), debe posibilitarse la descarga de idéntica información en un formato estándar que permita este tratamiento.

6. No discriminación: La información debe ser accesible a cualquiera, sin ningún tipo de requerimiento o registro previo. Debe posibilitarse el acceso de manera anónima a la información sin ningún tipo de condicionante.

7. No propietario: La información ha de estar disponible en formatos para los cuales ninguna entidad tenga control exclusivo. Los formatos propietarios añaden restricciones innecesarias sobre quién puede emplear los datos, cómo pueden ser usados y compartidos y si podrán ser accedidos en el futuro.

8. Licencias libres: La divulgación de la información no debe verse limitada por normas de propiedad intelectual, por cláusulas contractuales o por otros términos de carácter arbitrario. Si bien los marcos legales que en Estados democráticos regulan la privacidad de los ciudadanos y la seguridad pública pueden considerarse limitaciones aceptables, las restricciones a la divulgación por cualquier medio deben considerarse como la excepción y no como la norma, debiendo entregarse porciones de información cuando sea posible en informaciones restringidas.

9. Crowdsourcing: El mayor valor de la información deviene de su capacidad para ser accedida, comprendida y manejada por el público en general, cobrando entonces pleno sentido la función de divulgación.

10. Seguridad: La publicación de contenidos debe hacerse en formatos que no incluyan contenido ejecutable, dado el riesgo de seguridad para los usuarios si algún atacante malicioso consigue su infección con virus o troyanos.

11. Confianza: La información divulgada debe ser firmada electrónicamente e incluir testimonio de la fecha de creación o publicación, de su autenticidad y de su integridad. 
A partir de estos elementos, el objetivo, según detalla García (2014), es "lograr una gestión pública más transparente, colaborativa y participativa, que ofrezca soluciones más eficientes y acordes con los intereses de los ciudadanos" (p.86).

Como se puede desprender de lo anterior, existen diversos conceptos relacionados con el término de "transparencia", por lo que se ha considerado oportuno acuñar el concepto descrito por Martínez (2013), que establece que la transparencia es "la apertura del flujo de información [...] Que explica cómo las organizaciones públicas y actores gubernamentales se enfrentan cada vez más a las demandas de la sociedad para revelar información" (p. 311). Aunque esta definición es amplia, permite inferir de manera asertiva el concepto, por cuanto justamente lo que se persigue es que los ciudadanos tengan acceso a información clara, oportuna y accesible.

\section{Rendición de cuentas:}

La gestión de rendición de cuentas, o accountability, es definida por O’Donell (2001, p. 1) de la siguiente forma:

la existencia de agencias estatales que tienen autoridad legal y están fácticamente dispuestas y capacitadas (empowered) para emprender acciones que van desde el control rutinario hasta sanciones penales o incluso impeachment, en relación con actos u omisiones de otros agentes o agencias del estado que pueden, en principio o presuntamente, ser calificadas como ilícitos.

Por otra parte, este mismo autor habla de las agencias de balance y asignadas, refiriéndose al conjunto de instituciones que son creadas para ejercer controles mutuos y organizar el flujo de poder y autoridad en el gobierno y el Estado. Cuando lo anterior funciona de manera efectiva, se lleva a cabo una distribución del trabajo entre las organizaciones de mayor relevancia y, por ende, se facilita la implementación de los procesos de control.

Estas relaciones intraestatales se originan en una agencia y el accountability horizontal es ejercido por alguno de los poderes (Ejecutivo, Legislativo o Judicial), a los que las constituciones democráticas contemporáneas intentan balancear (O'Donell, 2001, p. 2).

Algunas debilidades de este proceso, según detalla O'Donell (2001), pueden radicar en respuestas reactivas o intermitentes, frente a transgresiones de otras instituciones. Este tipo de acciones tienden a generar conflictos entre las supremas instituciones estatales. "Estos conflictos se agudizan aún más cuando, en los regímenes presidencialistas, involucran poderes -Ejecutivo y Legislativo- que comparten legitimidad electoral" (p. 9).

Schedler (1999) agrega que la rendición de cuentas "abarca de manera genérica tres formas diferentes para prevenir y corregir abusos de poder; obliga al poder a abrirse a la inspección pública; lo fuerza a explicar y justificar sus actos, y lo supedita a la amenaza de sanciones" (p.13).

En términos de integralidad y suficiencia, se recomienda adoptar para este término el concepto de la CEPAL (s.f., párr.1) que indica lo siguiente:

la rendición de cuentas consiste en que existan normas, reglamentos y mecanismos que orienten el comportamiento de las autoridades electas y funcionarios en el ejercicio del poder público y el gasto de los recursos fiscales. Estas normas deben incluir los requisitos sobre que las decisiones sean plenamente motivadas y justificadas con toda la información puesta a disposición del público. Que exista protección para los denunciantes y mecanismos que permitan reaccionar a las revelaciones de fechorías e irregularidades. 
A partir de los elementos expuestos en la definición, el concepto adquiere mayor claridad en sus dimensiones y alcances, comprendiendo la relevancia que cumple la gestión de Gobierno abierto en la prevención y el combate de la corrupción.

\section{Participación ciudadana}

Uno de los principios del Gobierno abierto, tal como se ha referenciado previamente, es la participación ciudadana, dado que esta constituye el mecanismo para lograr un equilibrio en los pesos y contrapesos de la gestión pública y de la gestión política.

Además de lo que ya se ha señalado, se hace necesario identificar formas concretas para transformar la tradicional democracia representativa en participativa, con el fin de que la ciudadanía tenga una mayor injerencia en la gestión pública y en el régimen político. Lo anterior constituye un proceso social de inclusión de los actores sociales para influir directa o indirectamente en el desarrollo de la sociedad y sus sistemas, inclusión necesaria para garantizar la equidad entre quienes conforman dicha sociedad.

El énfasis en distintas formas de participación y la intensidad con la que esta se ejerce depende en gran medida del modelo de democracia que se quiere promover (Villarreal, 2009). Esta propuesta requiere que el debate público de ciudadanos y ciudadanas sea el centro de la toma de decisiones políticas legítimas. De acuerdo con lo que señala Villarreal (2009), en el intercambio de argumentos deben tomarse en cuenta no solo a los expertos, sino a todos los ciudadanos afectados por un asunto, y se confía en la capacidad de las personas de ser racionales, de comunicarse, de formular y entender argumentos. Esto permite generar procesos de colaboración en el control democrático de aquellas instituciones en las que se hace una toma efectiva de decisiones y no solamente en el marco electoral (Muñoz, 2004). De ahí que la participación deba ser entendida no solo como un proceso político, sino que también los procesos económicos, sociales y culturales son susceptibles a la intervención de los ciudadanos en la administración de la sociedad.

A partir de los supuestos descritos, habrá una real participación ciudadana si:

(1) los que diseñan las políticas y aquellos afectados por estas se involucran en una relación interpersonal; (2) hay aprendizaje para todos los sectores involucrados (interacción educativa), y (3) la interacción entre profesionales, agentes gubernamentales y otros permite a las comunidades sostener sus posiciones y recomendaciones en un espíritu de respeto y cooperación

Lo anterior permite el desarrollo de competencias individuales, promueve la organización y activación de individuos, grupos y comunidades, y facilita la transformación de los contextos compartidos por aquellos involucrados en el proceso, quienes también tienden a desarrollar un mayor sentido de control sobre sus vidas, según concuerdan varios autores (Ortiz-Torres, 1999; Lara, 2007; Rappaport, 1981). De esta manera, los cambios que se promueven a través de estos procesos participativos deberían permitir eventualmente el fortalecimiento de capital social y mayor acceso a recursos de todo tipo.

¿Cómo se articula o se hace efectiva esta participación? Es aquí donde radican los retos principales: cuándo se hace necesario precisar posibles estructuras de participación ciudadana en el espacio político.

Tal como se ha venido detallando, existen diversas definiciones para este término. No obstante, uno de los términos más completos es el acuñado por Baño (1998), que establece que la participación ciudadana es "una intervención de los particulares en actividades públicas en tanto portadores de intereses sociales" (p. 15). Aunque esta definición es amplia, permite inferir de manera asertiva el concepto, por cuanto justamente lo que se persigue es que los ciudadanos tengan acceso a participar propositivamente en la toma de decisiones gubernamentales. 


\section{CONCLUSIONES Y RECOMENDACIONES}

En términos generales se puede concluir que el modelo de Gobierno abierto aún no cuenta con una definición y procedimientos de implementación consensuados. No obstante, los lineamientos establecidos en el Open Government Partnership y la Carta Iberoamericana para un Gobierno Abierto, entre otros, sirven como punto de partida y como referente internacional.

De igual manera, es esencial que los Gobiernos cuenten con una estrategia definida para la implementación del modelo, con recursos administrativos y financieros; así como también el desarrollo e implementación de estrategias de comunicación que faciliten el acercamiento entre las instituciones, la ciudadanía y grupos organizados.

Lo anterior con el fin de incentivar una participación efectiva, que facilite espacios de cocreación de políticas públicas, basándose en principios e intereses comunes, en los cuales prevalezca la búsqueda del bien común.

No obstante, algunas discusiones epistemológicas sobre la participación ciudadana y los niveles de transparencia que deben ser implementados continúan teniendo asidero, dado que se ha venido planteando cómo estos elementos podrían entorpecer la gestión gubernamental, en términos de eficiencia y en términos rigurosidad técnica y política, si no se toman las previsiones pertinentes.

Es en razón de lo expuesto, algunos autores sugieren que no todos los temas deben ser abiertos al escrutinio público y la participación, siendo comprensible que temas de seguridad nacional, aspectos relativos a datos privados de empresas o personas, o bien, procesos legales en curso, requerirán de la discrecionalidad estatal para garantizar los derechos e integridad de las partes.

Tomando en consideración lo anterior, una recomendación que se deriva del análisis realizado es definir estratos de participación, promoviendo que exista un foro nacional de integración multipartita con los principales actores tales como academia, sociedad civil organizada, Gobierno y organizaciones no gubernamentales, en el seno del cual se discutan temas de alcance nacional que puedan tener un impacto directo en el bienestar general de la población, comprendiendo dentro de lo anterior reflexiones orientadas a impulsar una gestión más eficiente y oportuna del aparato estatal, lo que implicaría una incidencia directa en el bienestar de la ciudadanía.

Por otra parte, deben existir foros de discusión y análisis con los diversos grupos organizados, para escuchar sus propuestas, necesidades y expectativas, procurando la mayor conciliación de intereses posible; y, finalmente, se sugiere establecer mecanismos de consulta y atención para las personas usuarias, comprendiendo que estas pueden ser un elemento medular para la identificación de las deficiencias estatales, duplicidad de funciones, trámites innecesarios e incluso normas contradictorias, facilitando así identificar prioridades de acción orientadas a mejorar la eficiencia del aparato estatal. 


\section{REFERENCIAS}

Aucoin, P. (1990). Administrative Reform in PubIic Management: Paradigms, PrincipIes, Paradoxes and PenduIums. Governance: An International Journal Policy and Administration 3(2).

Barria, D., González, B. y Cisternas, C. (2017). La Literatura sobre Gobierno abierto en Español. Análisis sobre las dinámicas de producción y citación. Revista de Ciencias Sociales y Humanidades, 28, 22-42. Recuperado de http:// web.a.ebscohost.com.ezproxy.sibdi.ucr.ac.cr:2048/ehost/pdfviewer/pdfviewer? $\mathrm{vid}=5 \& \operatorname{sid}=30 \mathrm{be} 6 \mathrm{a} 57-4 \mathrm{f} 76-4 \mathrm{c} 7 \mathrm{~b}-$ 8151-777482010761\%40sessionmgr4007

Baño, R. (1998). Participación Ciudadana: Elementos Conceptuales. Santiago, Chile: FLACSO-Chile. Recuperado de http://flacsochile.org/biblioteca/pub/publicos/1998/libro/002297.pdf\#page=15

CEPAL. (s.f.). De Gobierno abierto a Estado abierto. Recuperado de https://biblioguias.cepal.org/EstadoAbierto/ rendicion

Conde, C. y Gutiérrez, M. (2014). La experiencia de la OCDE en la implementación de políticas de Gobierno abierto. El desafío de la etapa de implementación. Revista del CLAD Reforma y Democracia, 58, 237-256. Recuperado de http://www.redalyc.org/articulo.oa?id=357533690008

Cutberto, M., Rocha, D. y Martínez, C. (2015). Modelo de Gobierno abierto en América Latina. Paralelismo de las políticas públicas de transparencia y corrupción. Revista de Ciencias Sociales, 53, 85-103. Recuperado de http:// web.a.ebscohost.com.ezproxy.sibdi.ucr.ac.cr:2048/ehost/pdfviewer/pdfviewer?vid=2\&sid=56d49a9c-2ce6-46508a6b-389cc0521e80\%40sessionmgr4008

García, J. (2014). Gobierno abierto: Transparencia, participación y colaboración en las Administraciones Públicas. Revista de Ciencias Administrativas y Sociales, 24(54), pp 75-88. Recuperado de https://revistas.unal.edu.co/index. $\mathrm{php/innovar/article/view/46441/47951}$

Gómez, C y Gasco, M. (30 de octubre, 2012). Y ahora... Gobierno abierto: nuevos términos en la constante búsqueda por la transparencia y la rendición de cuentas. XVIII Congreso Internacional del CLAD sobre Reforma del Estado y de la Administración Pública, Cartagena, Colombia.

Instituto Interamericano de Derechos Humanos (2017). Diccionario Electoral de Capel. Recuperado de https:// www.iidh.ed.cr/capel/diccionario/example-assets/books/diccionario.pdf

Lara, M. (2007). Democracia Participativa, Privatización y Cambio Social. Disertación doctoral sometida a la Escuela Graduada de Psicología en Recinto de Río Piedras de la Universidad de Puerto Rico.

Martínez, F. (2013). ¿Qué transparencia requiere el Gobierno abierto? Instituto Tecnológico de Monterrey, México. Recuperado de http://www.revistadegestionpublica.cl/Vol_II_No_2/Marinez.pdf

Muñoz, B. (2004). Sobre el quiebre en la democracia participativa. Nómadas, Universidad Complutense, Madrid, enero-julio, 009, 2-17.

O’Donell, G. (2001). Accountability horizontal: la institucionalización legal de la desconfianza política. Revista Reflexión y Análisis Político, 7, 7-31. Recuperado de https://rescr.files.wordpress.com/2010/01/o-donnellguillermo-accountability-horizontal-la-institucionalizacion.pdf 
Ortiz-Torres, B. (1999). El empowerment como alternativa teórica para América Latina. Revista Interamericana de Psicología, 33(2), 49-65.

Ortiz-Torres, B. y Lara, M. (2010). Participación ciudadana para la promoción de una política social de equidad: Los Foros Ciudadanos en Puerto Rico. En Burgos, N.; Urizar de Alvarado, L.; Bojórquez de Grajeda, M. y Díaz Argueta, J. (eds.). Política Social y Trabajo Social: Hacia la Construcción de la Equidad. Guatemala: Universidad San Carlos.

Quintanilla, G. y Gil, J. (2016). Gobierno abierto y datos vinculados: conceptos, experiencias y lecciones con base en el caso mexicano. México. Recuperado de http://www.redalyc.org/articulo.oa?id=357546620003

Rappaport, J. (1981). In praise of paradox: A social policy of empowerment over prevention. American Journal Community Psychology, 9, 1-25.

Sandoval, R. (2015). Gobierno abierto y transparencia: construyendo un marco conceptual. Revista de Ciencias Sociales, Universidad Autónoma del Estado de México, 68, mayo-agosto, 203-227. Recuperado de http://www. redalyc.org/articulo.oa? id $=10536227008$

Schedler, A. (1999). Conceptualizing Accountability. En Schedler, A.; Diamond, L. y Plattner, M. F. (eds.). The Self-Restraining State: Power and Accountability in New Democracies. Boulder y Londres: Lynne Rienner Publishers, 13-28.

Valenzuela, R. y Bojórquez, J. (2014). Modelos de implementación del Gobierno abierto en México. Instituto Tabasqueño de Transparencia y Acceso a la Información Pública. Universidad Autónoma de México (UNAM). Recuperado de https://archivos.juridicas.unam.mx/www/bjv/libros/9/4016/9.pdf

Villarreal, M. (2009). Participación Ciudadana y Políticas Públicas. Décimo Certamen de Ensayo Político organizado por la Comisión Estatal Electoral de Nuevo León. Recuperado de https://www.ceenl.mx/educacion/ certamen_ensayo/decimo/MariaTeresaVillarrealMartinez.pdf 